\title{
Sparse Victory - A Large Scale Systematic Comparison of Count-Based and Prediction-Based Vectorizers for Text Classification
}

\author{
Rupak Chakraborty \\ Ashima Elhence \\ Kapil Arora \\ Adobe Inc, India \\ Adobe Inc, India \\ Adobe Inc, India \\ rupak97.4@gmail.com elhenceashima@gmail.com karora@adobe.com
}

\begin{abstract}
In this paper we study the performance of several text vectorization algorithms on a diverse collection of 73 publicly available datasets. Traditional sparse vectorizers like Tf-Idf and Feature Hashing have been systematically compared with the latest state of the art neural word embeddings like Word2Vec, GloVe, FastText and character embeddings like ELMo, Flair. We have carried out an extensive analysis of the performance of these vectorizers across different dimensions like classification metrics (.i.e. precision, recall, accuracy), dataset-size, and imbalanced data (in terms of the distribution of the number of class labels).

Our experiments reveal that the sparse vectorizers beat the neural word and character embedding models on 61 of the 73 datasets by an average margin of 3-5\% (in terms of macro fl score) and this performance is consistent across the different dimensions of comparison.
\end{abstract}

\section{Introduction}

The use of text vectorization for NLP applications has its roots in information retrieval and allied fields for measuring semantic similarity as enshrined by Jones (1972). Traditional methods for converting text into a fixed length vector include a bag of words representation (Zelling, 1954), where each word in the vocabulary is represented by a unique index, Tf-Idf builds upon this by weighting the frequency of each word by the inverse count of its document occurrence thereby mitigating the noise induced by Zipfian distribution of words in natural language. These vector space models are often referred to as sparse discrete representations owing to the large number of zeros that predominate their vector representations. Building on this foundation, research direction was aimed at generating continuous distributional semantics of text using factorization of word co-occurrence matrix as evinced in Latent Semantic Analysis (Dumais et. al, 1988, 2004). These SVD (Singular Value Decomposition) based approaches form the precursors of modern topic modeling (Blei et. al, 2003, 2002). Feature hashing often referred to as the hashing trick (analogy to the kernel trick) involves using a non-cryptographic hash function to convert text (i.e. word tokens) to a corresponding numerical representation, these representations are made to be uniformly distributed by including a secondary hashing function which alters the sign bit of the output of the first hashing function. These have been shown to have provable error bounds (Weinberger et al, 2009) and have been previously used for collaborative spam filtering and large scale multitask learning (Attenberg et al, 2009, Weinberger et al, 2009).

The use of the word neural word embeddings was first coined by the authors (Bengio et al, 2003) in their landmark paper which showed the efficacy of using hidden layer representations for measuring semantic similarity between words. Building upon this, it was further demonstrated by (Collobert et al, 2011) that unsupervised pre-training of word vectors preserved their syntactic and semantic similarities which lead to state of the art results on many downstream tasks. But it wasn't until introduction of Word2Vec (Mikolov et. al, 2013) that neural word embeddings became mainstream, this in a sense opened the flood gates of research into these models. GloVe (Pennington et. al, 2014) uses a log-bilinear regression model that combines the advantages of the two major model families in the literature - global matrix factorization and local context window methods. Enriching word vectors with subword information has proven to be effective as can be seen in fastText(Bojanowski et.al, 2017). Recent embedding models like ELMo 
(Peters et. al, 2018) use masked language modeling and textual entailment tasks to generate contextsensitive character-level representations. In the same vein, Flair embeddings (Akbik et. al, 2018) leverage the internal states of a trained character language model to produce a novel type of word embedding which the authors allude to as contextual string embedding. Moving from individual word representations to document and phrase level representation, we observe a less spectacular retinue of research work. Notable among these are Skip-Thought (Kiros et al, 2015) and InferSent (Conneau et al, 2017). Recently proposed Universal Sentence Encoder (Cer et. al, 2018) which uses multi-task transfer learning based on the transformer architecture (Vaswani et. al, 2017) to deliver promising results on several natural language inference tasks.

In light of these prolific advances made in the field of text vectorization, it becomes necessary to evaluate the different algorithms on downstream tasks and juxtapose their performance with the traditional non-neural counterparts. Existing evaluations (Baroni et. al, 2014) have only focused on the semantic aspect of these representations while ignoring tasks like text classification. Even when comparisons are made on benchmarks similar to the GLUE benchmark (Wang et. al, 2018), they are almost always made with state of art deep neural network based classifiers, the nonneural classifiers like Random Forests, SVMs and GradientBoosting are left out. To the best of our knowledge there is no existing research which comprehensively evaluates the performance of modern text vectorizers on text classification tasks, it is this research gap which we want to bridge in the present study. The main contributions of the paper are the following -1 . We have collected, curated and standardized a set of 73 different datasets which cover all aspects of text classification in particular and language modeling in general. 2. We have extensively analyzed the performance of neural vectorizers like Word2Vec, GloVe, FastText, ELMo and Flair on these datasets across many dimensions like dataset-size, class imbalance, classification metrics and juxtaposed it with their count-based non-neural alternatives like Feature Hashing and Tf-Idf. 3. We have also reported results on the performance of traditional ML classifiers, since our main aim is to study the efficacy of vectorization algorithms we haven't included any neural network based classifiers in the present study. 4. Our benchmark contains 73 datasets in comparison to GLUE which has only 10 , thereby making it more diverse and challenging. Finally, we have made our source $\operatorname{code}^{++}$, datasets ${ }^{* *}$ (including train and test splits), result files and all other necessary information publicly available so that, researchers can reproduce our results and further the progress in the field. While not central to the study we have also carried out an interpretation analysis on the predictions of these vectorizers by using model agnostic, locally interpretable explanations (Riberio et. al, 2016), the results are not included in the paper, however interested readers are encouraged to refer to Appendix A for more details.

The paper is organized as follows - Section 1 introduces the paper and gives an overview of the prior research work. Section-2 provides details of our datasets and the models used. Section-3 elucidates the approach we have taken for our experiments. Section-4 presents the results of our experiments, including an extensive analysis. Section- 5 concludes the paper and provides useful future research directions.

\section{Data \& Model Details}

We have collected the datasets from a variety of online sites like Kaggle, Crowdflower (now known as FigureEight), DataTurks, UCI repository and others. They have been grouped into 8 categories for ease of analysis, these are - emotion, sentiment, reviews, medical, general classification, news, spam-fake-hate-ironic and other. The general classification category set includes things like gender classification, website categorization weather and disaster detection from tweets etc. The other category set includes a set of language tasks like natural language inference, duplicate question detection, objectivity-subjectivity analysis which have been recast in a classification framework to promote uniformity. Details about the metadata of each category is present in table 1 . All the end tasks are different text categorizations ranging from classification of sentiments, emotions, news articles, reviews, gender, hate speech detection etc. All the datasets have been standardized in a common format, this format contains only two fields one for the text data other for the class label. Refer to Appendix B for necessary details about the data standardization process. As can be inferred from table 1, all the categories contain more than a 


\begin{tabular}{|c|c|c|c|}
\hline Category & \# Datasets & $\begin{array}{c}\text { \# Avg } \\
\text { Tokens }\end{array}$ & $\begin{array}{c}\text { \# Avg } \\
\text { Sentences }\end{array}$ \\
\hline Sentiment & 16 & $1.1 * 10^{8}$ & $1.2 * 10^{7}$ \\
\hline Emotion & 2 & $1.6 * 10^{7}$ & $2.1 * 10^{6}$ \\
\hline Reviews & 7 & $1.2 * 10^{9}$ & $2.8 * 10^{7}$ \\
\hline News & 8 & $3.3 * 10^{8}$ & $4.2 * 10^{7}$ \\
\hline $\begin{array}{c}\text { General } \\
\text { Classification }\end{array}$ & 17 & $1.9 * 10^{8}$ & $4.8 * 10^{6}$ \\
\hline $\begin{array}{c}\text { Spam-Fake- } \\
\text { Hate-Ironic }\end{array}$ & 10 & $8.6 * 10^{7}$ & $2.0 * 10^{6}$ \\
\hline Medical & 6 & $2.9 * 10^{8}$ & $1.8 * 10^{7}$ \\
\hline Other & 7 & $1.8 * 10^{8}$ & $1.4 * 10^{7}$ \\
\hline
\end{tabular}

Table 1: Details of datasets on a category basis million sentences on an average. Out of the 73 datasets 17 contain more than $50 \mathrm{~K}$ data samples, 39 contain less than $10 \mathrm{~K}$ data points and 17 contain between $10 \mathrm{~K}$ to $50 \mathrm{~K}$ rows. On a per category basis we observe that the general classification category contains the greatest number of datasets whose size is greater than $50 \mathrm{~K}$, while the sentiment category contains the maximum concentration of datasets of size less than $10 \mathrm{~K}$. To get an insight into the distribution of the number of rows per category refer to the box and whisker plot in figure 1, the yaxis contains the number of rows on a logarithmic scale (base 10).

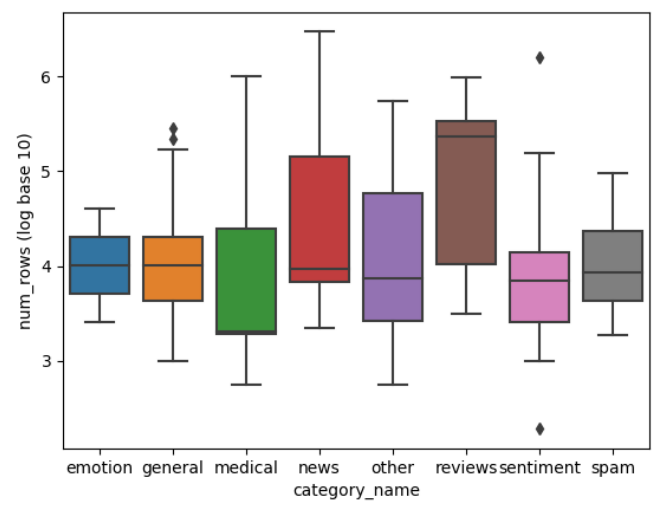

Figure 1: Box plot of distribution of rows per category

The neural embedding models have been pretrained** in the following way - the Word $2 \mathrm{Vec}$ model (of dimension 300) and has been trained on Google News Corpus (100 billion words). For greater ease of comparison both the GloVe and fastText models have a dimension of 300 and have been trained on the Common Crawl Corpus (640 billion words). The ELMo embedding has also been trained on Google News Corpus and as for the Flair embeddings we have used the original model provided by the authors which has been trained on English Wikipedia text. To provide a level-ground of comparison with the neural counterparts both the Tf-Idf and Feature Hashing vectorizers have a dimension of 300 . For the hashing vectorizer, a variant of Murmurhash3(Appleby, 2015) has been used to project the word tokens in a lower dimensional embedding space.

\section{Approach}

A systematic and comprehensive comparison of the vectorizers entails evaluating them across several dimensions, reporting the results using relevant metrics and then interpreting the results. The dimensions considered in the present study are the following -1 . Dataset-size, we consider 3 mutually exclusive and exhaustive ranges: less than $10 \mathrm{~K}$, greater than $50 \mathrm{~K}$ and between $10 \mathrm{~K}$ and $50 \mathrm{~K}$, these ranges have been chosen because they provide the most coverage across the selected datasets. For each of these ranges we analyze the performance on a per category basis. 2. Imbalance measure as reflected in the distribution of number of class labels (using equation 1).

Imbalance $=\sum_{\forall n 1, n 2} \frac{|n 1-n 2|}{n 1+n 2}$

In equation $1, \mathrm{n} 1$ and $\mathrm{n} 2$ are the number of samples belonging to classes $C_{i}$ and $C_{j}$. For a given dataset containing $\mathrm{N}$ different classes we find the ratio of the absolute difference of the number of datapoints in the two classes to the total number of data points in the two classes, we calculate this for all pairs of classes. For a perfectly balanced dataset this value will be zero, higher the value more will be the imbalance measure, there is no upper bound on the value. For the imbalance measure we calculate the terciles and divide the datasets into three parts based on the interval of these tercile values they are - $[0,1.03),[1.03,4.46),[4.46, \infty]$, where $\infty$ denotes the max value across all the datasets.

As mentioned in section 1, we have only considered non-neural classifiers as our main aim is to study the performance of vectorizers. The classifiers included in the present study are Random Forests, GradientBoost, AdaBoost, SVM (Linear Kernel) and Logistic Regression. These have been included because they represent a healthy mix of both bagging and boosting approaches along with linear models. For each dataset we measure its performance across all combinations of vectorizers and classifiers. 


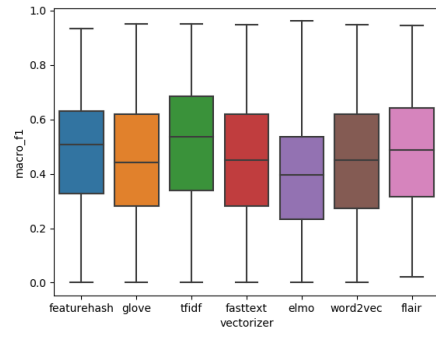

Figure 2: Vectorizer f1-score (global)

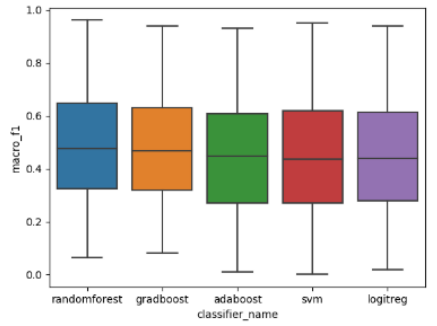

Figure 3: Classifier f1-score (global)

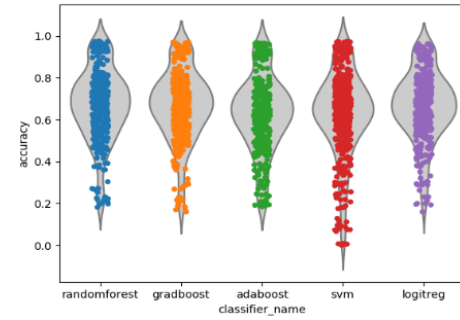

Figure 4: Classifier accuracy (global)

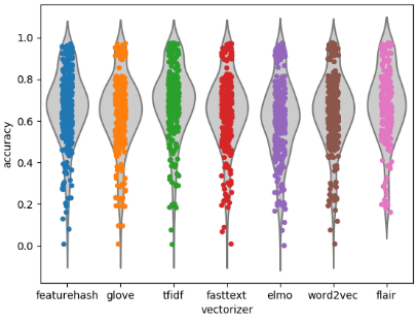

Figure 5: Vectorizer accuracy (global)

\section{Experiments \& Results}

All the experiments have been performed on a 32 GB Intel i7 processor, with a clock rate of 3.40 GHz. Since all the embedding models have been pre-trained, using a dedicated GPU doesn't result in a significant speedup, as we have noticed an increase of only $1.5 \mathrm{x}-2 \mathrm{x}$ while using a Nvidia GTX Geforce $1080 \mathrm{Ti}, 11 \mathrm{~GB}$ graphics processor. The total training time for all combinations of datasets, vectorizers and classifiers is more than 3 weeks.

We have carried out basic pre-processing of the text data like - case normalization, stopword removal, punctuation and special character removal followed by word tokenization, though it will be interesting to see the effects of more sophisticated pre-processing like lemmatization on the results. The hyperparameter settings of all the classifiers have been set to default values as used in the scikit-learn library except for number of trees in the Random Forest model which has been set to 51. Figures 2 and 3 provide a global view of the macro $\mathrm{fl}$ score of the vectorizers and classifiers averaged across all the datasets. For a given vectorizer we have calculated the mean performance metric (precision, recall, accuracy) across all classifiers and datasets. As can be inferred from figure 2, tf-idf and feature hashing consistently outperform their heavy weight neural counterparts, among the neural vectorizers flair embeddings demonstrate competitive performance on almost all datasets. The violin plots shown in figures 4 and 5 elucidate the performance of the classifiers and vectorizers (based on accuracy) under the same conditions as figures 2 and 3 . We can observe the same trends in these figures as we have previously seen in figures 2, 3. With respect to classifiers, Random Forests, Gradient Boost and Logistic Regression are always among the top performing trio. Apart from this, we have also seen that our results ${ }^{*}$ conform to widely established

trends like the negative correlation between the increase in number of classes and classifier performance metrics, we will expand upon this more in the section on analyzing performance metrics based on class imbalance.

\subsection{For Size Less Than 10K}

Tables 2 and 5, illustrate the performance of vectorizers and classifiers for all datasets whose size is less than $10 \mathrm{~K}$. The results have been grouped on a per category basis, in the category column the number inside the brackets denotes the number of datasets which fall into that category for the given dataset size range. The mean values of Precision Recall and Accuracy have been juxtaposed by following the notation Pr./Rec./Acc. We notice a wide variance in the performance metrics across the categories especially for reviews and emotion. The reason for this is that the emotion category has a dataset which has 18 classes while only containing 2524 samples, same is the case for reviews which has a dataset containing 41 classes. It is this small sample size and sparse data problem which reflects in the suboptimal performance of the vectorizers and classifiers. The number of class labels for all the other datasets in this size range lies between 2-5. Again, we observe that, tf-idf and feature hash come out on top consistently beating the neural counterparts (except for Flair) by a margin of 10\% (in terms of accuracy). On the classifier front again Random Forests, Gradient Boost and Logistic Regression edge out SVMs and AdaBoost. In context of the vectorizers we would like to make a case for feature hashing, extolling its many virtues which include - low computational footprint, the absence of a fixed vocabulary, theoretical error bounds and competitive performance, which serve to make it an ideal candidate for establishing strong baselines. 


\begin{tabular}{|c|c|c|c|c|c|c|c|}
\hline $\begin{array}{l}\text { Category } \\
\text { Name }\end{array}$ & $\begin{array}{c}\text { GloVe } \\
\text { (Pr./Rec./Acc.) } \\
\end{array}$ & $\begin{array}{c}\text { FastText } \\
\text { (Pr./Rec./Acc.) }\end{array}$ & $\begin{array}{c}\text { Word2Vec } \\
\text { (Pr./Rec./Acc.) }\end{array}$ & $\begin{array}{c}\text { ELMo } \\
\text { (Pr./Rec./Acc.) } \\
\end{array}$ & $\begin{array}{c}\text { Tf-Idf } \\
\text { (Pr./Rec./Acc.) } \\
\end{array}$ & $\begin{array}{l}\text { FeatureHash } \\
\text { (Pr/Rec./Acc.) }\end{array}$ & $\begin{array}{c}\text { Flair } \\
\text { (Pr/Rec./Acc.) } \\
\end{array}$ \\
\hline $\begin{array}{l}\text { Sentiment } \\
\text { (10) }\end{array}$ & $41.6 / 38.1 / 59.5$ & 42.9/38.9/59.9 & $42.9 / 38.2 / 59.4$ & $36.1 / 35.1 / 57.1$ & $47.0 / 42.2 / 63.3$ & 45.0/41.3/61.8 & $43.3 / 38.9 / 60.0$ \\
\hline Emotion (1) & $14.3 / 10.3 / 21.2$ & $12.5 / 9.1 / 20.4$ & $11.7 / 9.6 / 20.8$ & 7.9/7.0/19.0 & 14.2/10.2/19.1 & $15.0 / 10.6 / 18.3$ & $8.6 / 8.2 / 18.6$ \\
\hline $\begin{array}{c}\text { General } \\
\text { Classification } \\
(8)\end{array}$ & $56.8 / 49.5 / 64.8$ & $55.9 / 49.2 / 64.6$ & $54.3 / 48.6 / 64.0$ & $46.8 / 44.9 / 61.5$ & $60.7 / 55.3 / 68.3$ & $58.2 / 51.8 / 65.1$ & $56.5 / 52$ \\
\hline Other (5) & $59.7 / 56.8 / 67.8$ & $59.7 / 56.4 / 67.4$ & $59.1 / 56.6 / 67.6$ & $52.9 / 52.1 / 65.5$ & 61.5/55.6/69.8 & 57.1/53.3/68.6 & $59.1 / 52.8 / 67.0$ \\
\hline Reviews (2) & $52.1 / 37.6 / 83.4$ & $44.2 / 37.5 / 83.2$ & $52.1 / 37.6 / 83.2$ & $45.6 / 37.7 / 83.1$ & $57.4 / 43.9 / 85.4$ & $50.0 / 43.6 / 84.1$ & $55.8 / 42.2 / 84.0$ \\
\hline $\begin{array}{l}\text { Spam-Fake- } \\
\text { Ironic-Hate } \\
\text { (5) }\end{array}$ & 75.9/71.0/82.6 & 78.0/72.4/83.7 & 77.8/72.4/83.6 & 70.7/64.8/81.0 & $84.3 / 79.3 / 87.6$ & $80.0 / 74.9 / 84.5$ & 79.9/76.3/85.4 \\
\hline Medical (4) & $45.2 / 40.2 / 70.3$ & $42.9 / 40.3 / 70.1$ & $45.6 / 40.8 / 70.3$ & $40.6 / 36.9 / 68.7$ & 53.8/45.9/73.8 & $47.3 / 42.2 / 70.6$ & $49.3 / 42.2 / 71.3$ \\
\hline News (4) & $50.6 / 49.4 / 66.6$ & $48.6 / 48.3 / 66.2$ & $48.9 / 48.7 / 66.1$ & $35.9 / 36.6 / 54.3$ & 63.0/60.0/77.6 & $58.1 / 55.8 / 73.2$ & $63.2 / 60.9 / 78.4$ \\
\hline
\end{tabular}

Table 2. Mean Performance Metrics on a category basis for all Vectorizers (dataset size less than 10K)

\begin{tabular}{|c|c|c|c|c|c|c|c|}
\hline $\begin{array}{c}\text { Category } \\
\text { Name }\end{array}$ & $\begin{array}{c}\text { GloVe } \\
\text { (Pr./Rec./Acc.) }\end{array}$ & $\begin{array}{c}\text { FastText } \\
(\mathrm{Pr} / \text { Rec./Acc.) }\end{array}$ & $\begin{array}{c}\text { Word2Vec } \\
\text { (Pr/Rec./Acc.) }\end{array}$ & $\begin{array}{c}\text { ELMo } \\
\text { (Pr./Rec./Acc.) }\end{array}$ & $\begin{array}{c}\text { Tf-Idf } \\
\text { (Pr./Rec./Acc.) }\end{array}$ & $\begin{array}{l}\text { FeatureHash } \\
\text { (Pr./Rec./Acc.) }\end{array}$ & $\begin{array}{c}\text { Flair } \\
\text { (Pr./Rec./Acc.) }\end{array}$ \\
\hline Sentiment (4) & $54.5 / 45.5 / 60.8$ & $55.8 / 46.7 / 61.7$ & $55.5 / 46.4 / 61.5$ & $52.9 / 42.2 / 59.2$ & $64.0 / 57.0 / 68.6$ & $60.1 / 52.7 / 65.2$ & $57.6 / 49.9 / 63.0$ \\
\hline Emotion (1) & $14.9 / 11.9 / 27.5$ & $13.9 / 12.4 / 28.3$ & $14.2 / 12.3 / 28.0$ & $13.7 / 10.7 / 25.7$ & 23.1/16.0/31.5 & $15.8 / 13.8 / 28.4$ & $14.8 / 12.6 / 28.6$ \\
\hline $\begin{array}{c}\text { General } \\
\text { Classification } \\
\text { (6) }\end{array}$ & $47.4 / 41.9 / 58.9$ & $48.5 / 43.1 / 59.9$ & 48.8/42.9/59.6 & $41.8 / 37.6 / 54.7$ & $60.4 / 56.7 / 68.5$ & $57.4 / 52.0 / 65.1$ & $52.3 / 46.1 / 63.1$ \\
\hline Reviews (1) & $35.9 / 24.3 / 56.6$ & $33.9 / 24.3 / 56.6$ & $34.7 / 24.4 / 56.6$ & $30.9 / 23.1 / 54.9$ & $44.1 / 33.2 / 60.9$ & 43.4/29.6/58.7 & $36.1 / 25.2 / 55.4$ \\
\hline $\begin{array}{c}\text { Spam-Fake- } \\
\text { Ironic- } \\
\text { Hate(4) }\end{array}$ & $61.4 / 51.8 / 76.7$ & $63.4 / 53.2 / 77.6$ & $63.5 / 53.0 / 77.4$ & $58.5 / 47.4 / 74.8$ & $61.5 / 54.5 / 79.0$ & $60.7 / 51.4 / 76.9$ & $67.0 / 54.2 / 78.3$ \\
\hline News (1) & $15.9 / 9.2 / 50.5$ & $15.7 / 9.0 / 49.8$ & $16.2 / 9.5 / 51.7$ & 14.8/9.0/46.6 & $37.1 / 29.3 / 75.4$ & $44.9 / 36.7 / 74.6$ & $23.3 / 16.7 / 59.2$ \\
\hline
\end{tabular}

Table 3. Mean Performance Metrics on a category basis for all Vectorizers (dataset size between 10K and 50K)

\begin{tabular}{|c|c|c|c|c|c|c|c|}
\hline $\begin{array}{c}\text { Category } \\
\text { Name }\end{array}$ & $\begin{array}{c}\text { GloVe } \\
\text { (Pr/Rec./Acc.) }\end{array}$ & $\begin{array}{c}\text { FastText } \\
\text { (Pr./Rec./Acc.) }\end{array}$ & $\begin{array}{c}\text { Word2Vec } \\
\text { (Pr./Rec./Acc.) }\end{array}$ & $\begin{array}{c}\text { ELMo } \\
\text { (Pr./Rec./Acc.) } \\
\end{array}$ & $\begin{array}{c}\text { Tf-Idf } \\
\text { (Pr./Rec./Acc.) }\end{array}$ & $\begin{array}{l}\text { FeatureHash } \\
\text { (Pr/Rec./Acc.) }\end{array}$ & $\begin{array}{c}\text { Flair } \\
\text { (Pr./Rec./Acc.) }\end{array}$ \\
\hline Sentiment (2) & $58.5 / 52.0 / 61.7$ & $57.3 / 50.7 / 62.2$ & $55.6 / 50.5 / 61.9$ & $54.0 / 46.1 / 56.6$ & $56.0 / 48.6 / 59.3$ & $54.6 / 49.2 / 59.3$ & $64.1 / 55.2 / 62.0$ \\
\hline $\begin{array}{c}\text { General } \\
\text { Classification } \\
\text { (3) }\end{array}$ & $34.5 / 29.3 / 45.4$ & $34.9 / 30.6 / 45.6$ & $34.1 / 29.0 / 44.3$ & 29.0/26.7/42.6 & $34.6 / 31.7 / 46.0$ & $35.1 / 29.7 / 44.9$ & $34.4 / 29.8 / 44.5$ \\
\hline Other (2) & $53.7 / 48.2 / 59.6$ & $55.2 / 49.2 / 60.5$ & $54.9 / 49.2 / 60.5$ & $48.3 / 46.7 / 57.8$ & $48.3 / 44.4 / 54.0$ & $49.7 / 46.9 / 55.9$ & $54.3 / 47.2 / 57.6$ \\
\hline Reviews (4) & $33.7 / 22.0 / 44.0$ & $37.0 / 25.0 / 48.1$ & $34.8 / 24.2 / 45.8$ & $30.6 / 21.8 / 46.1$ & $38.0 / 28.4 / 54.0$ & $38.5 / 28.4 / 54.3$ & $37.2 / 27.4 / 52.2$ \\
\hline $\begin{array}{c}\text { Spam-Fake- } \\
\text { Ironic- } \\
\text { Hate(1) }\end{array}$ & $89.2 / 63.4 / 92.5$ & 90.5/65.9/93.0 & $90.8 / 64.8 / 92.5$ & $76.1 / 55.2 / 91.3$ & $82.1 / 62.6 / 92.3$ & $80.9 / 58.4 / 90.3$ & 83.0/63.5/91.7 \\
\hline Medical (2) & $64.4 / 61.7 / 68.5$ & $64.5 / 62.0 / 69.7$ & $62.0 / 59.9 / 69.5$ & $60.9 / 56.8 / 65.0$ & 67.3/65.5/70.1 & $64.7 / 62.9 / 70.2$ & 65.4/63.6/68.5 \\
\hline News (3) & $40.0 / 35.3 / 42.8$ & $42.2 / 38.1 / 44.4$ & $42.1 / 38.2 / 45.3$ & $36.8 / 29.3 / 34.0$ & $42.4 / 40.7 / 47.7$ & 42.0/39.4/46.1 & $41.7 / 37.7 / 47.6$ \\
\hline
\end{tabular}

Table 4. Mean Performance Metrics on a category basis for all Vectorizers (dataset size greater than 50K) 


\begin{tabular}{|c|c|c|c|c|c|}
\hline Category Name & $\begin{array}{l}\text { RandomForest } \\
\text { (Pr./Rec./Acc.) }\end{array}$ & $\begin{array}{l}\text { GradientBoost } \\
\text { (Pr./Rec./Acc.) }\end{array}$ & $\begin{array}{c}\text { AdaBoost } \\
\text { (Pr./Rec./Acc) }\end{array}$ & $\begin{array}{c}\text { Logit Regression } \\
\text { (Pr./Rec./Acc.) }\end{array}$ & $\begin{array}{l}\text { SVM (Linear) } \\
\text { (Pr./Rec./Acc.) }\end{array}$ \\
\hline Sentiment (10) & $46.5 / 40.2 / 60.8$ & $44.7 / 39.3 / 60.6$ & $38.4 / 37.4 / 57.7$ & $41.1 / 38.4 / 60.9$ & $42.7 / 39.4 / 60.7$ \\
\hline Emotion (1) & $15.9 / 11.2 / 20.5$ & $13.9 / 9.9 / 18.5$ & $5.4 / 6.2 / 19.2$ & $11.2 / 8.0 / 20.5$ & $14.0 / 11.3 / 19.5$ \\
\hline $\begin{array}{c}\text { General } \\
\text { Classification } \\
(8)\end{array}$ & $58.7 / 51.7 / 66.0$ & $58.2 / 52.0 / 66.2$ & $50.2 / 45.9 / 59.3$ & $53.8 / 50.3 / 66.4$ & $57.1 / 51.2 / 65.8$ \\
\hline Other (5) & 61.6/57.1/68.6 & $60.1 / 55.6 / 68.0$ & $58.0 / 54.8 / 66.2$ & $54.0 / 51.8 / 68.2$ & $58.2 / 55.0 / 67.6$ \\
\hline Reviews (2) & 69.8/51.9/87.1 & $64.1 / 47.6 / 85.8$ & $38.8 / 33.5 / 81.1$ & $36.8 / 30.9 / 82.3$ & $45.7 / 36.2 / 82.7$ \\
\hline $\begin{array}{l}\text { Spam-Fake- } \\
\text { Ironic-Hate (5) }\end{array}$ & $80.6 / 73.5 / 84.8$ & 80.8/73.9/85.0 & $76.1 / 73.4 / 82.7$ & $74.0 / 70.7 / 83.9$ & $78.2 / 73.6 / 84.0$ \\
\hline Medical (4) & 49.1/42.3/71.9 & $46.5 / 41.7 / 71.6$ & $42.9 / 39.8 / 67.7$ & $46.1 / 40.1 / 71.6$ & 47.4/42.0/71.0 \\
\hline News (4) & $53.6 / 51.6 / 69.5$ & $56.5 / 53.1 / 70.7$ & $47.9 / 46.8 / 63.4$ & $51.8 / 52.4 / 70.4$ & $53.3 / 52.7 / 70.5$ \\
\hline
\end{tabular}

Table 5. Mean Performance Metrics on a category basis for all Classifiers (dataset size less than 10K)

\begin{tabular}{|cccccc|}
\hline Category Name & $\begin{array}{c}\text { RandomForest } \\
\text { (Pr./Rec./Acc.) }\end{array}$ & $\begin{array}{c}\text { GradientBoost } \\
\text { (Pr./Rec./Acc.) }\end{array}$ & $\begin{array}{c}\text { AdaBoost } \\
\text { (Pr./Rec./Acc) }\end{array}$ & $\begin{array}{c}\text { Logit Regression } \\
\text { (Pr./Rec./Acc.) }\end{array}$ & $\begin{array}{c}\text { SVM (Linear) } \\
\text { (Pr./Rec./Acc.) }\end{array}$ \\
\hline Sentiment (4) & $\mathbf{5 8 . 7 / 4 8 . 3 / 6 2 . 8}$ & $\mathbf{5 9 . 1 / 4 7 . 5 / 6 3 . 0}$ & $52.6 / 46.8 / 60.5$ & $50.0 / 46.2 / 62.1$ & $\mathbf{5 7 . 6 / 4 8 . 5 / 6 3 . 1}$ \\
\hline Emotion (1) & $15.5 / 12.7 / 27.3$ & $16.9 / 13.6 / 29.1$ & $12.9 / 10.6 / 24.9$ & $\mathbf{1 8 . 1 / 1 3 . 5 / 2 9 . 8}$ & $\mathbf{1 6 . 4 / 1 3 . 9 / 3 0 . 2}$ \\
\hline $\begin{array}{c}\text { General } \\
\text { Classification } \\
(6)\end{array}$ & $\mathbf{5 4 . 9 / 4 6 . 3 / 6 1 . 9}$ & $51.6 / 46.8 / 62.1$ & $43.8 / 41.7 / 56.6$ & $49.9 / 46.2 / 62.1$ & $\mathbf{5 3 . 0 / 4 7 . 5 / 6 3 . 0}$ \\
\hline $\begin{array}{c}\text { Reviews (1) } \\
\text { (39.5/25.8/56.9 }\end{array}$ & $36.9 / 25.7 / 57.2$ & $35.2 / 26.8 / 56.6$ & $35.4 / 26.7 / 58.0$ & $\mathbf{3 8 . 8} / \mathbf{2 7 . 4 / 5 8 . 1}$ \\
\hline $\begin{array}{c}\text { Spam-Fake- } \\
\text { Ironic-Hate (4) }\end{array}$ & $\mathbf{7 6 . 9 / 6 0 . 1 / 8 2 . 9}$ & $65.1 / 52.2 / 76.6$ & $51.2 / 48.4 / 73.9$ & $55.7 / 48.6 / 75.8$ & $58.6 / 50.2 / 76.2$ \\
\hline News (1) & $\mathbf{4 1 . 5 / 2 2 . 8 / 6 4 . 9}$ & $27.5 / 21.6 / 59.8$ & $1.7 / 2.3 / 41.5$ & $21.6 / 17.2 / 60.8$ & $28.1 / 21.7 / 63.6$ \\
\hline
\end{tabular}

Table 6. Mean Performance Metrics on a category basis for all Classifiers (dataset size between 10K and 50K)

\begin{tabular}{|cccccc|}
\hline Category Name & $\begin{array}{c}\text { RandomForest } \\
\text { (Pr./Rec./Acc.) }\end{array}$ & $\begin{array}{c}\text { GradientBoost } \\
\text { (Pr./Rec./Acc.) }\end{array}$ & $\begin{array}{c}\text { AdaBoost } \\
\text { (Pr./Rec./Acc) }\end{array}$ & $\begin{array}{c}\text { Logit Regression } \\
\text { (Pr./Rec./Acc.) }\end{array}$ & $\begin{array}{c}\text { SVM (Linear) } \\
\text { (Pr./Rec./Acc.) }\end{array}$ \\
\hline Sentiment (2) & $\mathbf{5 7 . 1 / 5 2 . 3 / 6 1 . 4}$ & $58.0 / 46.8 / 60.1$ & $55.9 / 44.1 / 61.2$ & $\mathbf{6 2 . 4 / 6 2 . 5 / 6 2 . 4}$ & $53.3 / 47.0 / 58.8$ \\
\hline $\begin{array}{c}\text { General } \\
\text { Classification } \\
(3)\end{array}$ & $\mathbf{4 1 . 5 / 3 4 . 2 / 5 1 . 3}$ & $39.5 / 32.8 / 51.9$ & $38.5 / 31.4 / 45.9$ & $37.0 / 33.5 / 50.9$ & $23.2 / 22.2 / 32.5$ \\
\hline $\begin{array}{c}\text { Other (2) } \\
\text { Reviews (4) }\end{array}$ & $\mathbf{5 5 . 0 / 5 2 . 0 / 6 1 . 3}$ & $\mathbf{5 3 . 9} / \mathbf{4 7 . 4 / 5 8 . 8}$ & $48.8 / 45.2 / 54.7$ & $46.2 / 43.7 / 53.6$ & $48.4 / 44.4 / 55.6$ \\
\hline $\begin{array}{c}\text { Spam-Fake- } \\
\text { Ironic-Hate (1) }\end{array}$ & $\mathbf{8 9 . 7 / 6 2 . 1 / 9 2 . 3}$ & $\mathbf{8 8 . 8 / 6 3 . 7 / 9 2 . 5}$ & $84.0 / 64.5 / 92.0$ & $75.6 / 56.0 / 90.7$ & $25.7 / 20.2 / 43.3$ \\
\hline Medical (2) & $\mathbf{6 9 . 3 / 6 7 . 7 / 7 3 . 8}$ & $\mathbf{6 3 . 1 / 6 1 . 0 / 7 0 . 0}$ & $33.7 / 36.3 / 46.7$ & $59.7 / 57.0 / 65.4$ & $63.1 / 59.5 / 65.5$ \\
\hline News (3) & $48.8 / 43.1 / 50.8$ & $47.8 / 46.1 / 52.5$ & $46.2 / 45.0 / 51.4$ & $\mathbf{5 3 . 3 / 5 1 . 6 / 5 5 . 8}$ & $33.0 / 27.9 / / 34.6$ \\
\hline
\end{tabular}

Table 7. Mean Performance Metrics on a category basis for all Classifiers (dataset size greater than 50K) 


\begin{tabular}{|cccccccc|}
\hline $\begin{array}{c}\text { Category } \\
\text { Name }\end{array}$ & $\begin{array}{c}\text { GloVe } \\
\text { (Pr./Rec./Acc.) }\end{array}$ & $\begin{array}{c}\text { FastText } \\
\text { (Pr.Rec/Acc.) }\end{array}$ & $\begin{array}{c}\text { Word2Vec } \\
\text { (Pr./Rec./Acc.) }\end{array}$ & $\begin{array}{c}\text { ELMo } \\
\text { (Pr.Rec./Acc.) }\end{array}$ & $\begin{array}{c}\text { Tf-Idf } \\
\text { (Pr./Rec./Acc.) }\end{array}$ & $\begin{array}{c}\text { FeatureHash } \\
\text { (Pr/Rec./Acc.) }\end{array}$ & $\begin{array}{c}\text { Flair } \\
\text { (Pr./Rec./Acc.) }\end{array}$ \\
\hline Sentiment (4) & $69.0 / 69.0 / 69.0$ & $69.7 / 69.7 / 69.7$ & $69.4 / 69.3 / 69.3$ & $62.1 / 61.7 / 61.6$ & $\mathbf{7 4 . 9 / 7 4 . 1 / 7 4 . 1}$ & $\mathbf{7 1 . 7 / 7 1 . 4 / 7 1 . 4}$ & $69.0 / 68.2 / 68.2$ \\
\hline $\begin{array}{c}\text { General } \\
\text { Classification } \\
(7)\end{array}$ & $59.4 / 57.1 / 65.0$ & $60.5 / 58.5 / 66.1$ & $59.6 / 57.9 / 65.6$ & $51.4 / 52.0 / 60.8$ & $\mathbf{6 3 . 4 / 6 0 . 9 / 6 7 . 3}$ & $59.2 / 56.5 / 63.7$ & $\mathbf{6 1 . 2 / 6 1 . 2 / 6 7 . 5}$ \\
\hline Other (5) & $65.1 / 61.0 / 71.7$ & $65.5 / 61.1 / 71.7$ & $\mathbf{6 4 . 8 / 6 1 . 2 / 7 1 . 8}$ & $57.6 / 56.4 / 69.8$ & $\mathbf{6 5 . 2 / 5 8 . 4 / 7 1 . 8}$ & $62.2 / 57.3 / 71.5$ & $\mathbf{6 4 . 1 / 5 7 . 9 / 7 2 . 6}$ \\
\hline Reviews (1) & $80.3 / 54.8 / 91.9$ & $64.9 / 54.2 / 91.4$ & $79.2 / 54.3 / 91.6$ & $69.0 / 54.5 / 91.7$ & $\mathbf{8 2 . 6 / 5 8 . 1 / 9 2 . 0}$ & $73.8 / 63.1 / 91.5$ & $\mathbf{8 4 . 4 / 5 9 . 5 / 9 2 . 1}$ \\
\hline $\begin{array}{c}\text { Spam-Fake- } \\
\text { Ironic-Hate } \\
(8)\end{array}$ & $75.3 / 69.1 / 80.2$ & $76.7 / 70.7 / 80.9$ & $76.5 / 70.4 / 80.8$ & $69.6 / 63.2 / 77.7$ & $\mathbf{8 0 . 8 / 7 5 . 0 / 8 3 . 9}$ & $76.2 / 70.8 / 80.9$ & $77.2 / 72.8 / 81.7$ \\
\hline $\begin{array}{c}\text { Medical (2) } \\
72.2 / 65.9 / 84.6\end{array}$ & $69.1 / 65.3 / 87.0$ & $72.5 / 65.6 / 86.9$ & $63.4 / 62.0 / 85.9$ & $\mathbf{6 9 . 4 / 6 4 . 4 / 8 3 . 3}$ & $\mathbf{7 1 . 1 / 6 4 . 0 / 8 5 . 4}$ & $\mathbf{6 4 . 5 / 5 8 . 9 / 9 3 . 7}$ \\
\hline News (3) & $64.4 / 64.1 / 64.5$ & $62.9 / 62.6 / 63.2$ & $63.5 / 63.3 / 63.7$ & $43.3 / 41.6 / 42.4$ & $\mathbf{8 0 . 9 / 8 0 . 1 / 8 0 . 3}$ & $71.7 / 71.5 / 71.6$ & $\mathbf{8 3 . 8 / 8 3 . 6 / 8 3 . 8}$ \\
\hline
\end{tabular}

Table 8. Mean Performance Metrics on a category basis for all Vectorizers (for imbalance measure between 0.0 and 1.03)

\begin{tabular}{|cccccccc|}
\hline $\begin{array}{c}\text { Category } \\
\text { Name }\end{array}$ & $\begin{array}{c}\text { GloVe } \\
\text { (Pr./Rec./Acc.) }\end{array}$ & $\begin{array}{c}\text { FastText } \\
\text { (Pr/Rec./Acc.) }\end{array}$ & $\begin{array}{c}\text { Word2Vec } \\
\text { (Pr./Rec./Acc.) }\end{array}$ & $\begin{array}{c}\text { ELMo } \\
\text { (Pr./Rec./Acc.) }\end{array}$ & $\begin{array}{c}\text { Tf-Idf } \\
\text { (Pr./Rec./Acc.) }\end{array}$ & $\begin{array}{c}\text { FeatureHash } \\
\text { (Pr./Rec./Acc.) }\end{array}$ & $\begin{array}{c}\text { Flair } \\
\text { (Pr./Rec./Acc.) }\end{array}$ \\
\hline Sentiment (8) & $44.8 / 35.9 / 58.5$ & $46.9 / 36.9 / 59.2$ & $47.2 / 36.7 / 58.9$ & $41.5 / 33.7 / 58.1$ & $\mathbf{4 9 . 7 / 4 2 . 0 / 6 2 . 0}$ & $48.5 / 40.3 / 60.1$ & $49.4 / 39.5 / 60.4$ \\
\hline $\begin{array}{c}\text { General } \\
\text { Classification } \\
(6)\end{array}$ & $51.1 / 42.9 / 60.9$ & $49.6 / 42.5 / 60.6$ & $48.0 / 41.8 / 60.1$ & $42.4 / 38.9 / 57.7$ & $\mathbf{5 3 . 9 / 4 8 . 7 / 6 5 . 6}$ & $52.2 / 45.8 / 62.2$ & $\mathbf{5 2 . 8 / 4 7 . 3 / 6 3 . 2}$ \\
\hline Other (2) & $\mathbf{4 0 . 0 / 3 8 . 0 / 5 0 . 1}$ & $39.5 / 37.7 / 49.9$ & $\mathbf{3 9 . 6 / 3 8 . 1 / 5 0 . 4}$ & $36.0 / 35.6 / 47.6$ & $\mathbf{3 9 . 9 / 3 8 . 2 / 5 0 . 4}$ & $37.0 / 37.1 / 49.4$ & $\mathbf{4 4 . 3 / 3 7 . 5 / 5 0 . 2}$ \\
\hline Reviews (2) & $36.0 / 24.2 / 37.5$ & $36.2 / 25.9 / 35.9$ & $37.9 / 25.7 / 34.2$ & $29.4 / 22.2 / 36.2$ & $35.9 / 31.6 / 41.2$ & $\mathbf{3 7 . 9 / 3 1 . 7 / 4 2 . 0}$ & $36.3 / 30.2 / 39.6$ \\
\hline $\begin{array}{c}\text { Spam-Fake- } \\
\text { Ironic-Hate } \\
(1)\end{array}$ & $62.0 / 42.6 / 79.2$ & $61.9 / 44.6 / 79.8$ & $63.2 / 44.1 / 79.9$ & $63.4 / 38.5 / 77.7$ & $\mathbf{7 0 . 5 / 6 3 . 8 / 8 8 . 0}$ & $67.2 / 55.0 / 84.7$ & $67.9 / 43.4 / 79.5$ \\
\hline $\begin{array}{c}\text { Medical (1) } \\
54.2 / 54.0 / 57.8\end{array}$ & $55.8 / 55.8 / 55.7$ & $56.4 / 56.3 / 56.4$ & $52.7 / 48.0 / 52.6$ & $\mathbf{6 7 . 7 / 6 4 . 8 / 6 5 . 8}$ & $61.7 / 61.9 / 62.0$ & $59.5 / 59.5 / 59.6$ \\
\hline News (2) & $38.9 / 37.0 / 70.8$ & $36.6 / 36.4 / 71.4$ & $36.8 / 36.5 / 70.7$ & $32.7 / 34.3 / 68.7$ & $\mathbf{4 3 . 9 / 3 9 . 1 / 7 3 . 9}$ & $\mathbf{4 2 . 3 / 3 7 . 8 / 7 2 . 6}$ & $\mathbf{4 3 . 1 / 3 8 . 7 / 7 3 . 5}$ \\
\hline
\end{tabular}

Table 9. Mean Performance Metrics on a category basis for all Vectorizers (for imbalance measure between 1.03 and 4.46)

\begin{tabular}{|c|c|c|c|c|c|c|c|}
\hline $\begin{array}{c}\text { Category } \\
\text { Name }\end{array}$ & $\begin{array}{c}\text { GloVe } \\
\text { (Pr./Rec./Acc.) }\end{array}$ & $\begin{array}{c}\text { FastText } \\
\text { (Pr./Rec./Acc.) }\end{array}$ & $\begin{array}{c}\text { Word2Vec } \\
\text { (Pr./Rec./Acc.) }\end{array}$ & $\begin{array}{c}\text { ELMo } \\
\text { (Pr./Rec./Acc.) }\end{array}$ & $\begin{array}{c}\text { Tf-Idf } \\
\text { (Pr./Rec./Acc.) }\end{array}$ & $\begin{array}{l}\text { FeatureHash } \\
\text { (Pr/Rec./Acc.) }\end{array}$ & $\begin{array}{c}\text { Flair } \\
\text { (Pr./Rec./Acc.) }\end{array}$ \\
\hline Sentiment (4) & $26.5 / 24.1 / 54.1$ & $26.1 / 25.2 / 54.3$ & $25.0 / 23.4 / 53.8$ & $22.6 / 23.1 / 52.6$ & $33.8 / 27.9 / 59.2$ & $29.6 / 27.3 / 58.1$ & $23.8 / 22.6 / 54.0$ \\
\hline Emotion (2) & $14.6 / 11.1 / 24.4$ & $13.2 / 10.7 / 24.3$ & $13.1 / 11.0 / 24.4$ & $10.8 / 8.9 / 22.3$ & $18.7 / 13.1 / 25.3$ & $15.4 / 12.3 / 23.3$ & $12.1 / 10.5 / 22.9$ \\
\hline $\begin{array}{c}\text { General } \\
\text { Classification } \\
\text { (3) }\end{array}$ & $32.4 / 19.4 / 52.1$ & $32.8 / 19.7 / 51.8$ & $35.9 / 20.6 / 53.1$ & $27.8 / 17.8 / 50.8$ & $55.7 / 47.5 / 67.7$ & $52.6 / 40.4 / 63.5$ & 40.7/23.4/53.8 \\
\hline Reviews (4) & $30.0 / 19.3 / 56.2$ & 29.3/21.0/60.3 & $27.6 / 20.8 / 60.1$ & $24.5 / 20.3 / 59.6$ & $36.4 / 27.8 / 65.8$ & $33.7 / 24.7 / 63.3$ & $31.7 / 25.0 / 61.4$ \\
\hline $\begin{array}{l}\text { Spam-Fake- } \\
\text { Ironic-Hate } \\
\text { (1) }\end{array}$ & $47.1 / 31.7 / 89.3$ & $53.0 / 32.5 / 90.2$ & $52.6 / 33.4 / 90.1$ & 41.0/28.0/90.6 & $32.5 / 16.5 / 85.6$ & 41.0/19.8/87.0 & 58.8/34.9/91.1 \\
\hline Medical (3) & $35.6 / 31.0 / 64.5$ & $34.2 / 31.3 / 65.4$ & $34.6 / 30.5 / 65.4$ & $33.4 / 28.4 / 62.7$ & $46.5 / 38.4 / 68.5$ & $36.8 / 33.1 / 63.9$ & $36.6 / 25.3 / 66.0$ \\
\hline News (3) & $15.9 / 8.4 / 35.8$ & $15.8 / 8.3 / 36.3$ & $15.7 / 8.4 / 38.2$ & $14.6 / 7.0 / 34.6$ & $25.4 / 20.1 / 49.5$ & 30.8/24.2/49.8 & $25.0 / 17.0 / 54.8$ \\
\hline
\end{tabular}

Table 10. Mean Performance Metrics on a category basis for all Vectorizers (for imbalance measure between 4.46 and $\infty$ ) 


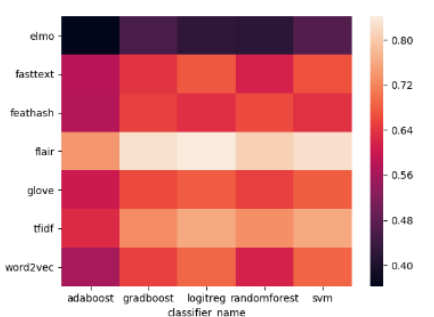

Figure 6: Heatmap(accuracy) for a news classification dataset

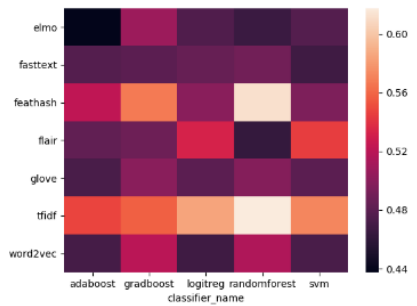

Figure 7: Heatmap(accuracy) for a inference dataset

\subsection{For Size Between $10 \mathrm{~K}-50 \mathrm{~K}$}

Tables 3 and 6 provide an overview of the performance metrics of the vectorizers and classifiers under the same conditions as tables 2 and 5 the only difference is that the range of datasets is between $10 \mathrm{~K}$ and $50 \mathrm{~K}$, since Medical and Other categories have no datasets in this range, they haven't been included in the tables. The trends observed here are consistent with the ones we have observed in section 4.1. The average number of class labels in this range is less than 10 for all categories except for news, which has a dataset containing 75 classes and emotion where the number of class labels is 13 for a given dataset.

\subsection{For Size Greater Than 50K}

Tables 4 and 7 illustrate the performance metrics for 17 datasets whose size is greater than $50 \mathrm{~K}$, the emotion category is missing because it has no datasets in this range. The presence of a dataset with 27995 class labels in the general classification category skews the results and leads to the observed performance metrics, same is the case for news which has a dataset containing 756 classes. For all the experiments we have used a train-test split of 80-20, the seed used for random split is kept same so the results will be consistent while reproducing. Again, the trends noticed here are faithful to the observed trends in section 4.1 and 4.2. Here we would briefly like to mention that our intention is not to undermine the spectacular advances of deep learning and state of the art results it has produced in NLP, we are aware of the fact that only deep models are capable of scaling in performance with the increase in data-size, however it might seem like an overkill in situations where simpler models do an equally good job. Refer to figure 6 (dataset used is of a news classification task), for a case where a neural embedding (Flair) beats every other non-neural and neural counterpart (in terms of accuracy). Figure 7 also illustrates an accuracy heatmap where the results are more in tune with the general trends of the study, the dataset used here is one of agreement-disagreement between sentence pairs.

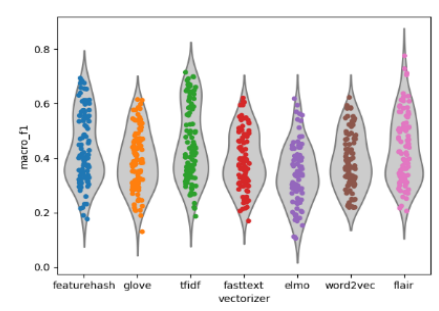

Figure 8: Performance (f1-score) for imbalance range $[1.03,4.46)$

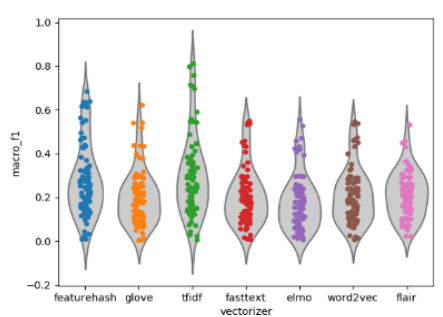

Figure 9: Performance (f1-score) for imbalance range $[4.46, \infty)$

\subsection{Metrics Under Class Imbalance}

Tables 8, 9 and10 demonstrate the performance of the vectorizers across the three selected strata of imbalance measure. Tf-Idf and Feature Hash shines in all three cases. As a general trend we have noticed that the accuracy of the vectorizers increase when the text is more verbose (i.e. news) in comparison to limited character content. (i.e. tweets). Flair embeddings show a competitive performance (when the imbalance measure is less than 4.46), sometimes even outperforming the sparse vectorizers, as per the results aggregated it is clearly our neural vectorizer of choice. The violin plots in figures 8 and 9, illustrate the performance of the vectorizers (macro fl score averaged across all datasets and classifiers) under different ranges of class imbalance (as calculated using equation 1). In all these cases feature hashing has the highest median performance as can inferred from the greatest density of points in the center. The tf-idf vectorizer has the highest variance in its performance because of a skew in class distribution, which in turn skews the performance of this count-based vectorizer. Word2Vec, FastText and GloVe have almost similar performance.

\section{Conclusion \& Future Work}

"Neural Embedding models are not a silver bullet", it is in this spirit that we have carried out the present study and reported the results. The analysis presented here might serve as a starting point for researchers new to this field, who might be overwhelmed by the plethora of alternate text vectorizers available. It will also serve as a strong baseline for future research direction in the domain of text classification. In the future we would like to use neural classifiers (CNNs and RNNs) in place of traditional ones presently used, so that we can provide a complete picture of classifier performance across the entire spectrum. Finetuning models like ELMo, ULMFit (Howard and Ruder, 2018) and observing the change in results will be an interesting line of future work. Additionally, we would also like to explore other embedding models like BERT (Devlin et. al, 2018) and CoVe (McCann et.al, 2017). 


\section{References}

Karen Sparck Jones. 1972. A statistical interpretation of term specificity and its application in retrieval. Journal of documentation 28(1):11-2.

Zellig S Harris. "Distributional structure. Word, 10 (23): 146-162. Reprinted in Fodor, J. A and Katz, JJ." Readings in the Philosophy of Language (1954): 33-49.

Susan T Dumais. "Latent semantic analysis." Annual review of information science and technology 38 , no. 1 (2004): 188-230

Susan T Dumais, George W. Furnas, Thomas K. Landauer, Scott Deerwester, and Richard Harshman. "Using latent semantic analysis to improve access to textual information." In Proceedings of the SIGCHI conference on Human factors in computing systems, pp. 281-285. Acm, 1988.

David M. Blei, Andrew Y. Ng, and Michael I. Jordan. "Latent dirichlet allocation." Journal of machine Learning research 3, no. Jan (2003): 993-1022.

David M. Blei, Andrew Y. Ng, and Michael I. Jordan. "Latent dirichlet allocation." In Advances in neural information processing systems, pp. 601-608. 2002.

Josh Attenberg, Kilian Weinberger, Anirban Dasgupta, Alex Smola, and Martin Zinkevich. "Collaborative email-spam filtering with the hashing trick." In Proceedings of the Sixth Conference on Email and Anti-Spam. 2009.

Kilian Weinberger, Anirban Dasgupta, Josh Attenberg, John Langford, and Alex Smola. "Feature hashing for large scale multitask learning." arXiv preprint arXiv:0902.2206 (2009).

Ronan Collobert, Jason Weston, Léon Bottou, Michael Karlen, Koray Kavukcuoglu, and Pavel Kuksa. "Natural language processing (almost) from scratch." Journal of machine learning research 12, no. Aug (2011): 2493-2537.

Yoshua Bengio, Réjean Ducharme, Pascal Vincent, and Christian Jauvin. "A neural probabilistic language model." Journal of machine learning research 3, no. Feb (2003): 1137-1155.

Tomas Mikolov, Ilya Sutskever, Kai Chen, Greg S. Corrado, and Jeff Dean. "Distributed representations of words and phrases and their compositionality." In Advances in neural information processing systems, pp. 3111-3119. 2013.

Jeffrey Pennington, Richard Socher, and Christopher Manning. "Glove: Global vectors for word representation." In Proceedings of the 2014 conference on empirical methods in natural language processing (EMNLP), pp. 1532-1543. 2014.

Piotr Bojanowski, Edouard Grave, Armand Joulin, and Tomas Mikolov. "Enriching word vectors with subword information." Transactions of the Association for Computational Linguistics5 (2017): 135-146.

Ryan Kiros, Yukun Zhu, Ruslan R. Salakhutdinov, Richard Zemel, Raquel Urtasun, Antonio Torralba, and Sanja Fidler. "Skip-thought vectors." In Advances in neural information processing systems, pp. 3294-3302. 2015.

Alexis Conneau, Douwe Kiela, Holger Schwenk, Loic Barrault, and Antoine Bordes. "Supervised learning of universal sentence representations from natural language inference data." arXiv preprint arXiv:1705.02364 (2017).

Daniel Cer, Yinfei Yang, Sheng-yi Kong, Nan Hua, Nicole Limtiaco, Rhomni St John, Noah Constant et al. "Universal sentence encoder." arXiv preprint arXiv:1803.11175 (2018)

Matthew E Peters, Mark Neumann, Mohit Iyyer, Matt Gardner, Christopher Clark, Kenton Lee, and Luke Zettlemoyer. "Deep contextualized word representations." arXiv arXiv:1802.05365 (2018).

Alan Akbik, Duncan Blythe, and Roland Vollgraf. "Contextual string embeddings for sequence labeling." In Proceedings of the 27th International Conference on Computational Linguistics, pp. 1638-1649. 2018.

Ashish Vaswani, Noam Shazeer, Niki Parmar, Jakob Uszkoreit, Llion Jones, Aidan N. Gomez, Łukasz Kaiser, and Illia Polosukhin. "Attention is all you need." In Advances in Neural Information Processing Systems, pp. 5998-6008. 2017.

Marco Baroni, Georgiana Dinu, and Germán Kruszewski. "Dont count, predict! A systematic comparison of context-counting vs. contextpredicting semantic vectors." In Proceedings of the 52nd Annual Meeting of the Association for Computational Linguistics (Volume 1: Long Papers), vol. 1, pp. 238-247. 2014.

Alex Wang, Amanpreet Singh, Julian Michael, Felix Hill, Omer Levy, and Samuel R. Bowman. "Glue: A multi-task benchmark and analysis platform for natural language understanding." arXiv preprint arXiv:1804.07461 (2018).

Marco Tulio Ribeiro, Sameer Singh, and Carlos Guestrin. "Why should i trust you?: Explaining the predictions of any classifier." In Proceedings of the 22nd ACM SIGKDD international conference on knowledge discovery and data mining, pp. 11351144. ACM, 2016. 
Austin Appleby. Murmurhash3 64-bit finalizer. Version 19/02/15. https://code. google. com/p/smhasher/wiki/MurmurHash3.

Jeremy Howard and Sebastian Ruder. "Universal Language Model Fine-tuning for Text Classification." In Proceedings of the 56th Annual Meeting of the Association for Computational Linguistics (Volume 1: Long Papers), vol. 1, pp. 328-339. 2018.

Jacob Devlin, Ming-Wei Chang, Kenton Lee, and Kristina Toutanova. "Bert: Pre-training of deep bidirectional transformers for language understanding." arXivpreprint arXiv:1810.04805 (2018).

Bryan McCann, James Bradbury, Caiming Xiong, and Richard Socher. "Learned in translation: Contextualized word vectors." In Advances in Neural Information Processing Systems, pp. 62946305. 2017.

Ajay Patel, Alexander Sands, Chris Callison-Burch, and Marianna Apidianaki. "Magnitude: A Fast, Efficient Universal Vector Embedding Utility Package." In Proceedings of the 2018 Conference on Empirical Methods in Natural Language Processing: System Demonstrations, pp. 120-126. 2018. 